\title{
Signal-Amplified Near-Infrared Ratiometric
}

\section{Electrochemiluminescence Aptasensor Based on}

\section{Multiple Quenching and Enhancement Effect of}

\section{Graphene/Gold Nanorods/G-quadruplex}

Kang Shao , Biru Wang ${ }^{\dagger}$, Shiyi $\mathrm{Ye}^{\dagger}$, Yunpeng Zuo ${ }^{\dagger}$, Long $\mathrm{Wu}^{\dagger}$, Qin $\mathrm{Li}^{\dagger}$, Zhicheng $\mathrm{Lu}^{\dagger}$, Xue Cai Tan

$$
{ }^{\ddagger}, \text { Heyou Han }{ }^{\mathrm{a}, \uparrow}
$$

${ }^{\dagger}$ State Key Laboratory of Agricultural Microbiology, College of Science, College of Food Science and Technology, Huazhong Agricultural University, Wuhan 430070, People’s Republic of China

ॠ School of Chemistry and Chemical Engineering, Guangxi University for Nationalities, Nanning 530008, People's Republic of China

* E-mail: hyhan@mail.hzau.edu.cn. Phone: +86-27-87288505. Fax: +86-27-87288505. 


\section{EXPERIMENTAL DETAIL}

\section{Preparation of NIR-emitting CdTe/CdS Core small $_{\text {Shell }}$ thick QDs}

MPA-capped NIR-emitting CdTe/CdS core $_{\text {small }} /$ shell $_{\text {thick }}$ QDs were directly synthesized in aqueous solution according to the previous method. ${ }^{1}$ Briefly, $2.5 \times 10^{-4}$ mol of $\mathrm{CdCl}_{2} \cdot 2.5 \mathrm{H}_{2} \mathrm{O}$ was dissolved in 50 $\mathrm{mL}$ of ultrapure water, and $37 \mu \mathrm{L}$ of MPA was added under stirring, followed by adjusting the $\mathrm{pH}$ to 12.2 by adding dropwise $1.0 \mathrm{M} \mathrm{NaOH}$. The solution was deaerated by $\mathrm{N}_{2}$ bubbling for 30 min. Under vigorous stirring, the freshly prepared NaHTe solution $\left(1.0 \times 10^{-5} \mathrm{~mol}\right)$ was injected to the deaerated solution. Next, the solution was aged at $4{ }^{\circ} \mathrm{C}$ overnight and the small CdTe cluster solution was obtained. The NIR-emitting CdTe/CdS coresmall/shell thick QDs were synthesized by further aging the small CdTe cluster solution at $90{ }^{\circ} \mathrm{C}$ for $10 \mathrm{~h}$.

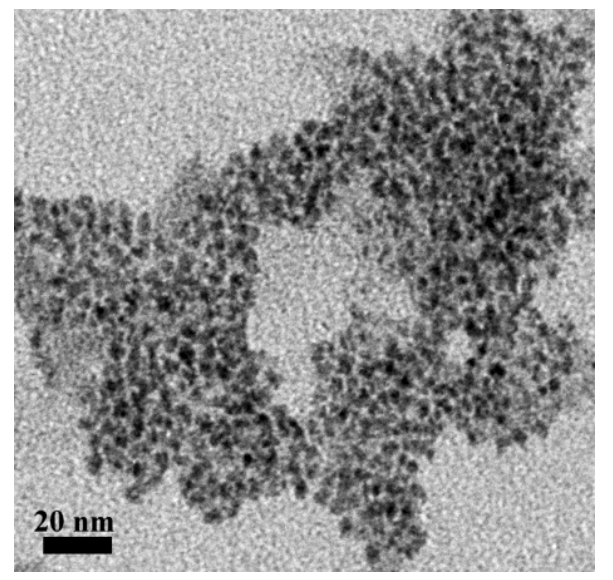

Figure S1. TEM image of CdTe/CdS.

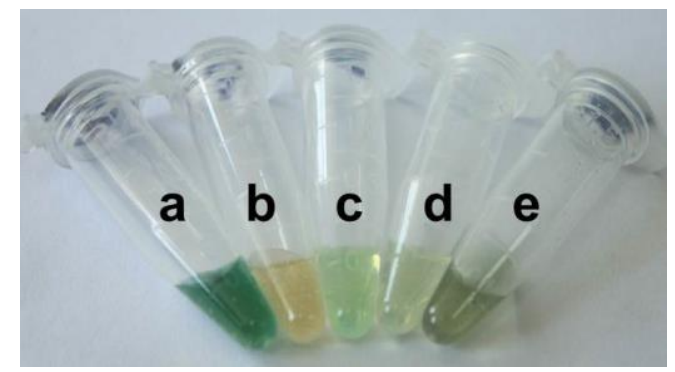

Figure S2. The colorimetric analysis of $\mathrm{TMB}-\mathrm{H}_{2} \mathrm{O}_{2}$ after adding the equal (a) rGO-H-AuNRs, (b) AuNRs, (c) hemin, (d) rGO and (e) rGO-H dispersions. 
The Possible Mechanism of the Dual-potential NIR-ECL Ratiometric Strategy

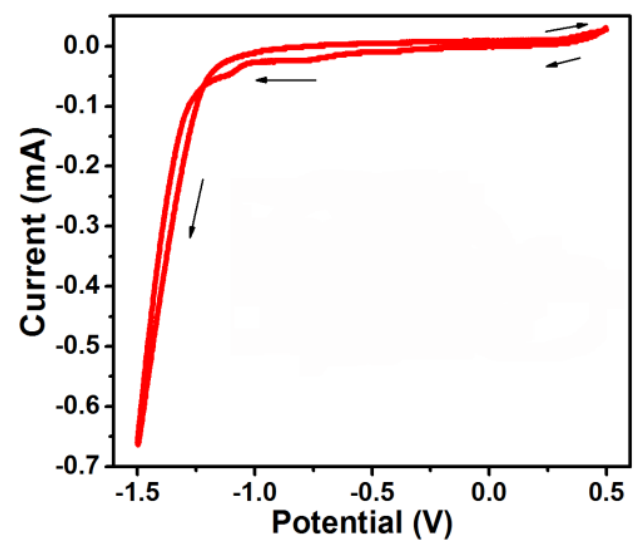

$$
\mathrm{H}_{2} \mathrm{O}_{2} \underset{+\mathrm{H}^{+}}{\stackrel{-\mathrm{H}^{+}}{\rightleftharpoons}} \mathrm{HO}_{2}^{-} \stackrel{-\mathrm{e}}{\longrightarrow} \mathrm{HO}_{2} \underset{+\mathrm{H}^{+}}{\stackrel{-\mathrm{H}^{+}}{\rightleftharpoons}} \mathrm{O}_{2}^{--}
$$

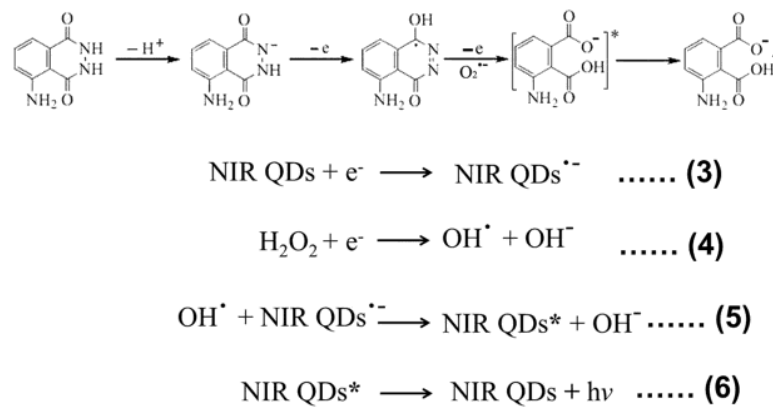

Figure S3. The possible mechanism of the dual-potential ECL ratiometric strategy

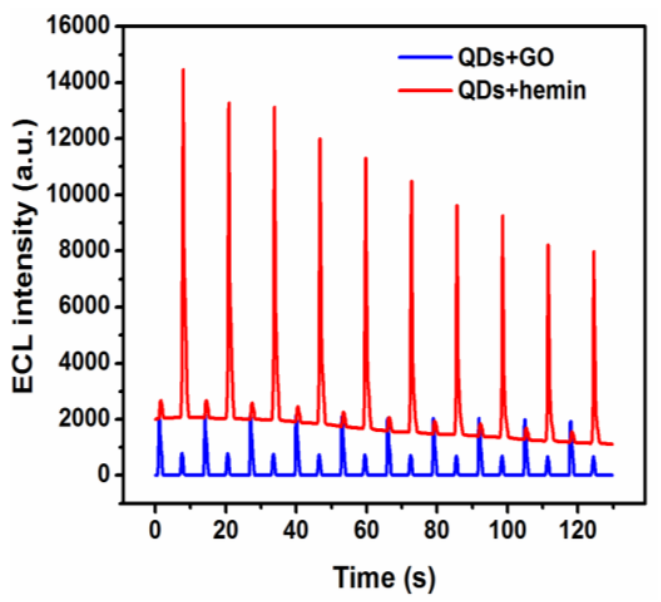

Figure S4. The ECL responses of QDs/GCE in different luminol PBS buffers containing GO (blue line) and hemin (red line). 

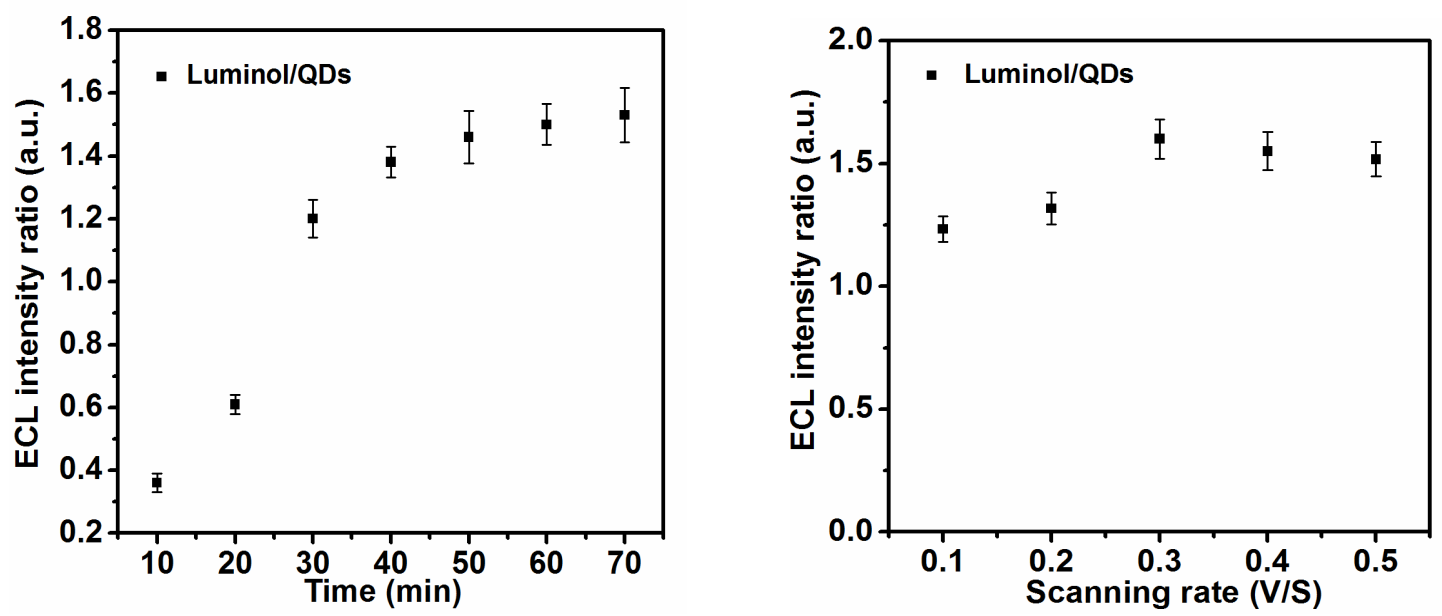

Figure S5. The optimization experiment of ECL biosensors with different incubation time and scanning rate.

Table S1. Comparison of the proposed NECL aptasensor with some other ECL aptasensor in the visible range for thrombin

\begin{tabular}{cccc}
\hline Detection methods & $\begin{array}{c}\text { Linear range } \\
(\mathrm{pM})\end{array}$ & $\begin{array}{c}\text { Detection limit } \\
(\mathrm{pM})\end{array}$ & Reference \\
\hline ECL & $10^{3} \sim 1.5 \times 10^{5}$ & 100 & {$[2]$} \\
ECL & $5.0 \sim 5.0 \times 10^{5}$ & 1.7 & {$[3]$} \\
ECL & $5.0 \sim 5.0 \times 10^{5}$ & 1.7 & {$[4]$} \\
ECL & $1.0 \sim 5.0 \times 10^{3}$ & 0.12 & {$[5]$} \\
ECL & $1.0 \sim 10^{4}$ & 0.1 & {$[6]$} \\
ECL & $0.1 \sim 10^{3}$ & 0.05 & {$[7]$} \\
ECL & $10^{-3} \sim 0.1$ & $10^{-3}$ & {$[8]$} \\
NIR ECL & $0.03 \sim 1.5 \times 10^{3}$ & $1.1 \times 10^{-4}$ & This work \\
ECL & $10^{-4} \sim 0.1$ & $2.6 \times 10^{-5}$ & {$[9]$} \\
ECL & $5.0 \times 10^{-6} \sim 5.0 \times 10^{-3}$ & $1.0 \times 10^{-6}$ & {$[10]$} \\
\hline
\end{tabular}

\section{REFERENCES}

(1) Deng, Z., Schulz, O., Lin, S., Ding, B., Liu, X., Wei, X., Ros, R., Yan, H., Liu, Y., 2010. J. Am. Chem. Soc.132, 5592-5593.

(2) Zhao P., Zhou L., Nie Z., Xu X., Li W., Huang Y., He K., Yao S., 2013. Anal. Chem. 85, 6279-6286.

(3) Li F., Cui H., 2013. Biosens. Bioelectron. 39, 261-267. 
(4) Liao Y., Yuan R., Chai Y., Mao L., Zhuo, Y. Yuan Y., Bai L., Yuan S., 2011. Sensor. Actuat. B: Chem. 158, 393-399.

(5) Jie G., Yuan J., 2012. Anal. Chem. 84, 2811-2817.

(6) Chen Q., Chen H., Zhao Y., Zhang F., Yang F., Tang J., He P., 2014. Biosens. Bioelectron. 54, 547552.

(7) Yang L., Zhu J., Xu Y., Yun W., Zhang R., He P., Fang Y., 2011. Electroanalysis 23, 1007-1012.

(8) Guo Y., Jia X., Zhang S., 2011. Chem. Commun. 47, 725-727.

(9) Wang J., Shan Y., Zhao W.-W., Xu J.-J., Chen H.-Y., 2011. Anal. Chem. 83, 4004-4011.

(10) Shan Y., Xu J.-J., Chen H.-Y., 2011, Nanoscale 3, 2916-2923. 